ARTICLE OPEN

Check for updates

\title{
Automated quantification of Candida albicans biofilm-related phenotypes reveals additive contributions to biofilm production
}

\author{
Matthew J. Dunn ${ }^{1,4}$, Robert J. Fillinger ${ }^{2,4}$, Leah M. Anderson ${ }^{1}$ and Matthew Z. Anderson $\mathbb{D}^{1,3 凶}$
}

Biofilms are organized communities of microbial cells that promote persistence among bacterial and fungal species. Biofilm formation by host-associated Candida species of fungi occurs on both tissue surfaces and implanted devices, contributing to host colonization and disease. In C. albicans, biofilms are built sequentially by adherence of yeast to a surface, invasion into the substrate, the formation of aerial hyphal projections, and the secretion of extracellular matrix. Measurement of these biofilm-related phenotypes remains highly qualitative and often subjective. Here, we designed an informatics pipeline for quantifying filamentation, adhesion, and invasion of Candida species on solid agar media and utilized this approach to determine the importance of these component phenotypes to C. albicans biofilm production. Characterization of 23 C. albicans clinical isolates across three media and two temperatures revealed a wide range of phenotypic responses among isolates in any single condition. Media profoundly altered all biofilm-related phenotypes among these isolates, whereas temperature minimally impacted these traits. Importantly, the extent of biofilm formation correlated significantly with the additive score for its component phenotypes under some conditions, experimentally linking the strength of each component to biofilm mass. In addition, the response of the genome reference strain, SC5314, across these conditions was an extreme outlier compared to all other strains, suggesting it may not be representative of the species. Taken together, development of a high-throughput, unbiased approach to quantifying Candida biofilm-related phenotypes linked variability in these phenotypes to biofilm production and can facilitate genetic dissection of these critical processes to pathogenesis in the host.

npj Biofilms and Microbiomes (2020)6:36; https://doi.org/10.1038/s41522-020-00149-5

\section{INTRODUCTION}

Formation of complex microbial communities, or biofilms, promotes organismal survival by resisting stress and providing a sheltered replicative niche for bacterial, archaeal, and eukaryotic microbes ${ }^{1}$. These structured communities of microbes are embedded within an extracellular matrix that stabilizes the local environment and limits exposure of cells to destructive elements including desiccation, UV radiation, and heavy metals ${ }^{2-5}$. In mammalian hosts, microbial biofilms also provide protection from clearance by host phagocytes and confer resistance to antimicrobial compounds ${ }^{6-9}$. Microbial persistence within human hosts due to biofilm formation is thought to be associated with approximately $80 \%$ of human infections caused by bacterial and fungal species $^{10-14}$

Among fungi, Candida species are common members of the human microflora where they reside within the oral, digestive, and genitourinary tracts of the host, as well as on the surface of the $\operatorname{skin}^{15,16}$. However, fungal overgrowth of these commensal niches can lead to debilitating superficial infections of the mucosa and skin, as well as systemic or deep-tissue infections that are associated with high mortality rates approaching $50 \%{ }^{17,18}$. These infections are particularly common in patients with implanted medical devices, including prosthetics, heart valves, dentures, and catheters, which are susceptible to colonization and biofilm formation by Candida species. Patients with catheter-associated biofilms are particularly vulnerable to fungal dissemination and systemic disease because the cells released from the biofilm have immediate access to the host circulatory system ${ }^{19-21}$. Among Candida species, C. albicans is most commonly isolated from colonized medical devices, although many Candida species can form clinically-relevant biofilms on both host biotic surfaces and the abiotic surfaces of implanted devices ${ }^{22}$.

Extensive work has detailed the morphological progression of biofilm formation in C. albicans. This highly structured biofilm requires the contribution of both yeast and hyphal cell morphologies ${ }^{23-25}$ and reaches maturity through four major sequential stages that can be observed both in vitro and in vivo ${ }^{23,26,27}$. First, yeast cells adhere to a surface via adhesins (e.g., EAP1 and the ALS gene family) that bind to a broad range of substrates and host ligands ${ }^{28-31}$. Following adherence, yeast cells initiate hyphal production, invading into the substrate and projecting aerially. Extracellular matrix composed primarily of glycoproteins is secreted during establishment of this complex, interwoven hyphal meshwork that encapsulates the entire community ${ }^{32}$. Finally, yeast-like disperser cells are released from aerial hyphae protruding through the extracellular matrix of the mature biofilm that are primed to establish new biofilms at distant locations, leading to disseminated disease ${ }^{25,28,33}$.

Nearly all studies of C. albicans biofilm-associated phenotypes have been performed in the genome reference strain, SC5314, with only a few investigations including other clinical isolate backgrounds ${ }^{34-36}$. Recently, key differences in the genetic regulation of biofilm production across C. albicans strain

\footnotetext{
${ }^{1}$ Department of Microbiology, The Ohio State University, Columbus, OH 43210, USA. ${ }^{2}$ Biomedical Sciences Graduate Program, The Ohio State University, Columbus, OH 43210, USA. ${ }^{3}$ Department of Microbial Infection and Immunity, The Ohio State University, Columbus, OH 43210, USA. ${ }^{4}$ These authors contributed equally: Matthew J. Dunn, Robert J. Fillinger. ${ }^{\circledR}$ email: anderson.3196@osu.edu
} 
backgrounds have suggested that current understandings of biofilm production may be fairly limited ${ }^{34}$. Indeed, analysis of strain collections have revealed significant variation in biofilm and filamentation phenotypes among isolates ${ }^{36,37}$.

Both solid and liquid substrate models have been used to study the biofilm-associated phenotypes of adhesion, filamentation, and invasion in C. albicans ${ }^{33,38-40}$. The classical method for simultaneously assaying these phenotypes uses a solid agar substrate to allow filamentation of $C$. albicans colonies, followed by rinsing the agar surface to assess colony adherence, and then physical removal of adherent colonies to reveal invasion profiles into the agar substrate. However, the genetic and environmental regulation of these biofilm-associated traits is often investigated without including their impact on biofilm formation ${ }^{38,41,42}$. Consequently, the relative importance of each process to establishment and maturation of biofilms remains somewhat obscure, although it is clear that mutants defective in adhesion or filamentation produce weak and stunted biofilms $\mathrm{s}^{43-45}$.

A major hurdle to dissecting biofilm-associated traits has been the lack of quantitative methods available to score each component phenotype. Most mutant studies for adhesion, filamentation, and invasion have relied on visual representations, descriptive language, and other qualitative measures. However, the introduction of the morphology $(\mathrm{M})$ score for agar-based plate assays vastly improved quantitative assessment of colony filamentation ${ }^{46}$. The $M$ score assigns each strain a relative score on a scale from -3 to +3 for center and peripheral colony filamentation, indicating decreased or increased filamentation, respectively, where a reference strain is set to zero. Subsequent adjustments to the $M$ score provided an absolute scale for assigning filamentation but retained numerous caveats (unequal weighting of hyphal production, time-intensive analysis, and use of a colony subset for scoring) and did not include other biofilmassociated phenotypes such as adhesion or invasion ${ }^{33,37}$. Screening adhesion phenotypes using high-throughput methods has also contributed to defining the genes that promote this process but relied on relative quantification within mutant pools, similar to the $\mathrm{M}$ score ${ }^{47,48}$.

Here, we describe an automated approach to quantify biofilmassociated phenotypes including adhesion, invasion, and filamentation using the canonical agar-based assay. Application of this method to a collection of 23 characterized C. albicans clinical isolates revealed significant variation in adhesion, invasion, and filamentation on solid media. Media but not temperature was the primary determinant of isolate phenotypes across strain backgrounds. Furthermore, the cumulative strengths of adhesion, filamentation, and invasion correlated with biofilm production under some conditions, suggesting a predictive model of biofilm formation exists based on its component phenotypes. Finally, the genome reference strain, SC5314, was a clear outlier among assayed strains across phenotypes, suggesting that current understanding of biofilm-related phenotypes and biofilm formation may yet be limited to the genome reference strain and less applicable across $C$. albicans isolates.

\section{RESULTS}

Previous investigation of filamentation on Spider medium and biofilm formation across a characterized set of 21 C. albicans clinical isolates revealed wide phenotypic breadth between strains $^{37}$. Consequently, we centered this study on those 21 isolates and two additional strains, $529 \mathrm{~L}^{49,50}$ and WO- $1^{51-53}$, which have noteworthy relevance to $C$. albicans pathogenesis and longstanding historical use. Together, these strains are a representative collection of isolates with significant genetic diversity to investigate variation in adhesion, invasion, and filamentation and their relative contributions to biofilm production.

Development of an automated pipeline for quantitative biofilmrelated phenotyping

To assess biofilm-associated phenotypes among C. albicans strains, we used the common agar plate-based assay that simultaneously allows measurement of colony filamentation, adhesion, and invasion of the substrate ${ }^{54}$. Each isolate was plated to 100 colonies on three solid agar media: Lee's, Spider, and YPD, and allowed to grow at either $30^{\circ} \mathrm{C}$ or $37^{\circ} \mathrm{C}$ for seven days. These media are ordered by increasing nutrient content: Lee's media contains amino acids as the primary carbon source, Spider provides primarily carbohydrates for growth, and YPD (yeast extract, peptone, dextrose) is a rich complete media.

After seven days of growth, plates were imaged from above, and the resultant images were interrogated with custom scripts to detect radial filaments (Fig. 1a, cyan and Supplemental Methods). The degree of radial filamentation was determined as $\left(\right.$ area $_{\text {hyphal growth }}-$ area $\left._{\text {center colonies }}\right) /\left(\right.$ area $\left._{\text {center colonies }}\right)$ to produce an absolute measurement of filamentation that includes all hyphal projections at pixel resolution. Filamentation within the central colony or 'wrinkling' was also measured by quantifying colony roughness along a gradient from the smooth, domed appearance characteristic of yeast-dominant colonies to a progressively wrinkled morphology due to the increased prevalence of hyphae (Fig. 1a, magenta). Following acquisition of this first image, the agar plate was washed with a constant stream of water to remove
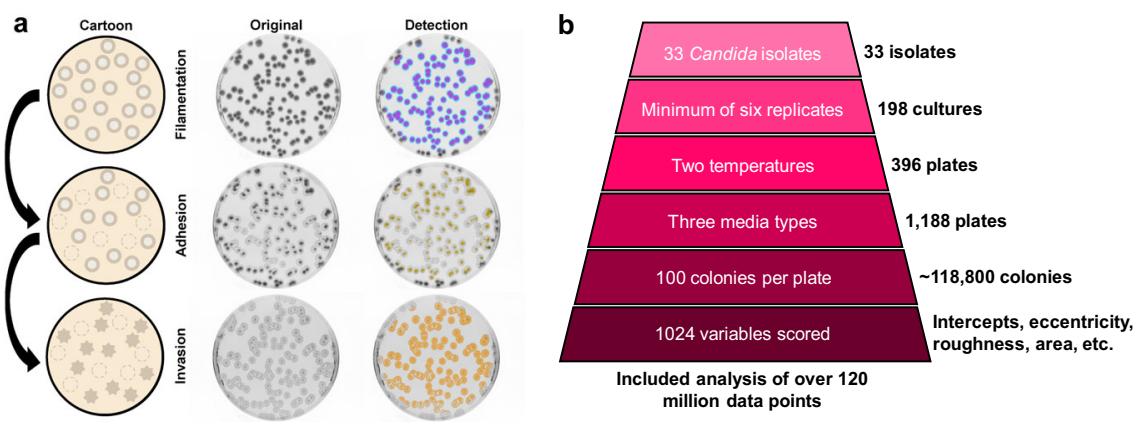

Fig. 1 Experimental approach for determining biofilm-related phenotypes. a One hundred cells from each strain were plated, allowed to grow for seven days, and the resulting colonies were imaged for filamentation (radial in cyan and center colony wrinkling in magenta). Plates were then rinsed with a stream of water and imaged for adhesion (yellow). Remaining colonies were wiped off and the plates imaged for agar invasion (orange). A cartoon, raw image, and detection overlay (left to right) has been shown to highlight this process. b Automated quantification facilitated measurement of $\sim 118,800$ colonies to collect over $120,000,000$ data points (C. albicans $-\sim 85$ million, non-albicans Candida species - 35 million) for analysis of filamentation, wrinkling, adhesion, and invasion. 

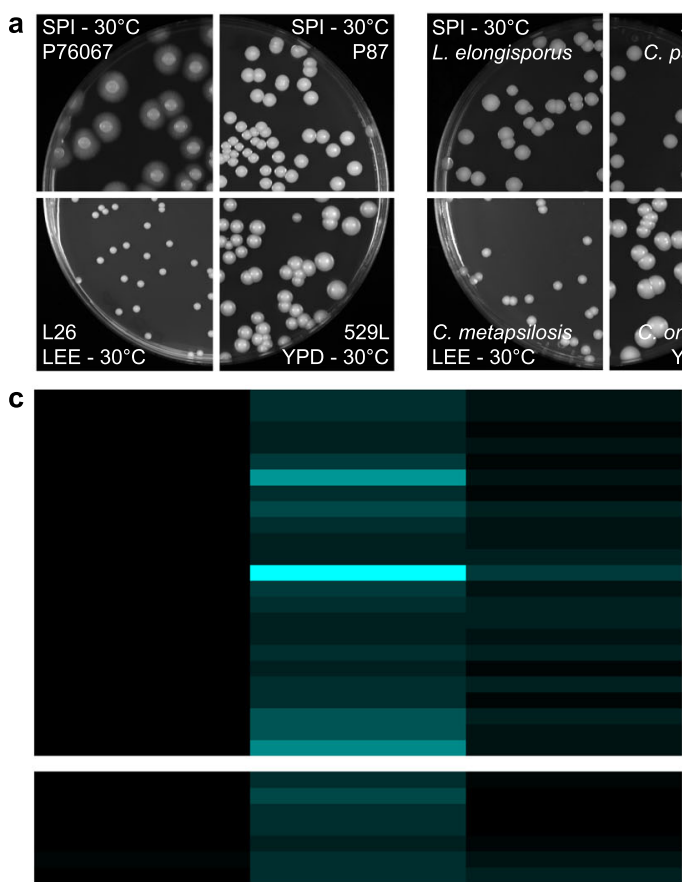

ш

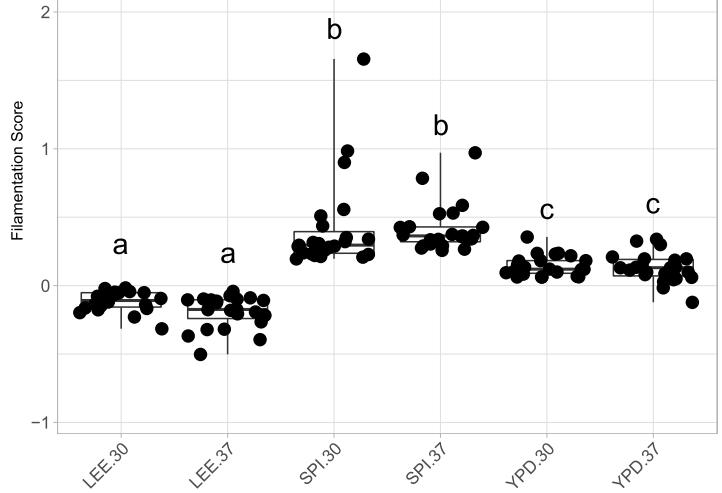

b
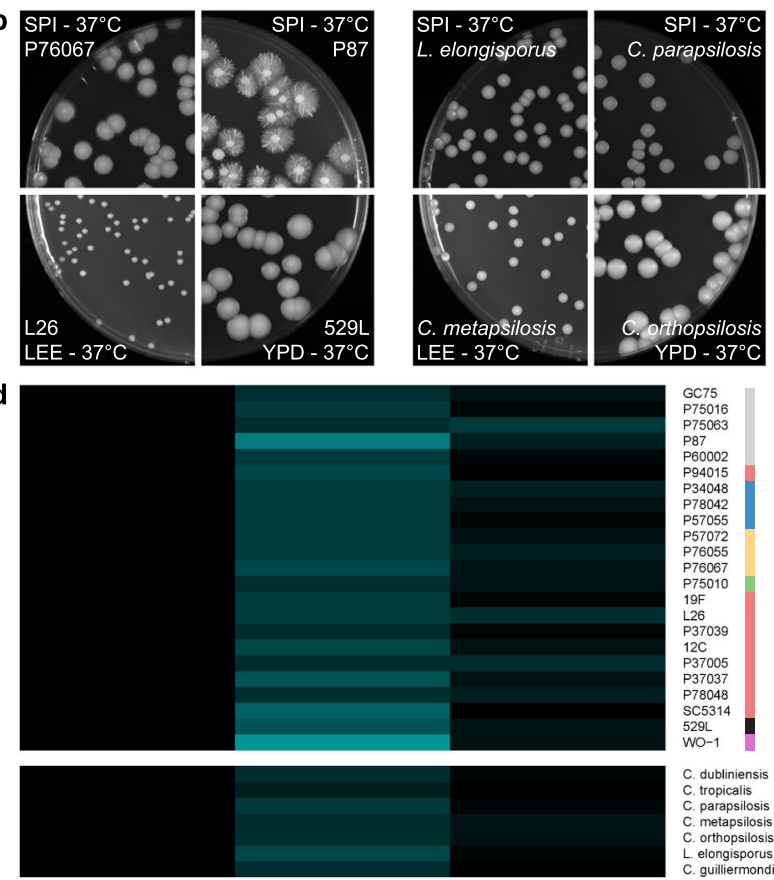

Ш

$\bar{c}$

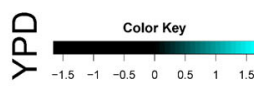

f PCA - Biplot

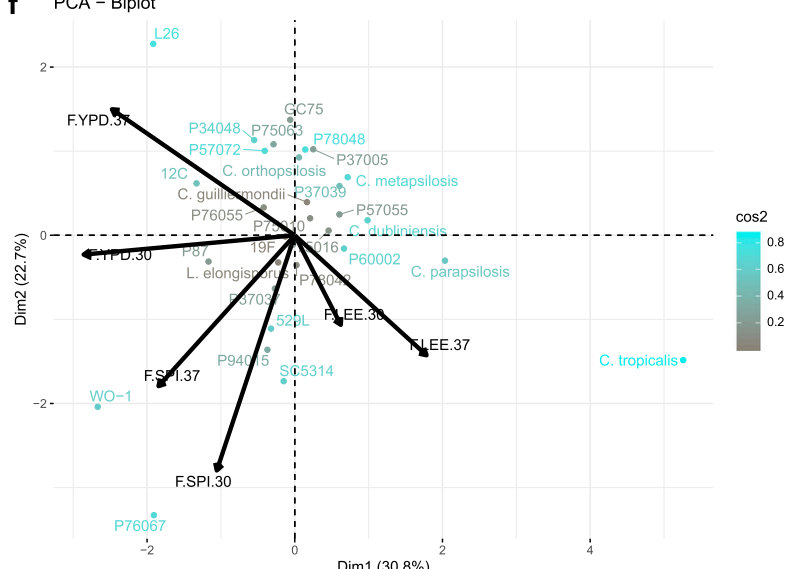

Fig. 2 Candida radial filamentation is largely defined by medium. Plate images of $C$. albicans isolates (left) and non-albicans Candida species (NACS) (right) highlight the varied radial filamentation profiles across media at $30^{\circ} \mathrm{C}$ (a) and $37^{\circ} \mathrm{C}$ (b). Heatmaps represent the degree of radial filamentation for Candida isolates at $30^{\circ} \mathrm{C}$ (c) and $37^{\circ} \mathrm{C}$ (d). Scoring for radial filamentation was defined by (area hyphal growth - area ${ }_{\text {center colonies }}$ )/ $\left(\right.$ area $\left._{\text {center colonies }}\right)$ for at least six biological replicates and averaged. Isolate clade is color coded on the right as in Hirakawa et al. ${ }^{37}$. e Radial filamentation scores for each C. albicans isolate were grouped and plotted for each media and temperature combination. Overlaid box plots cover the upper and lower interquartile ranges with the intervening line indicating the mean. Whiskers extend to the extreme datapoints. Letters indicate statistically different groups based on Bonferroni-corrected $p<0.05$ by Dunn's test. f PCA biplots were constructed using radial filamentation data for all assayed Candida isolates. Coloration from gray to cyan represents the power of the strain in defining PC1 and PC2. Black arrows represent vectorized phenotypic contributions where the arrows are labeled (Phenotype.Media.Temperature). LEE $=$ Lee's medium, SPI = Spider medium, YPD = Yeast Peptone Dextrose medium.

non-adherent colonies, allowed to dry, and then reimaged to measure isolate adherence (Fig. 1a, yellow). Adhesion was calculated as the $\left(\right.$ area $\left._{\text {colonies post-wash }}\right) /\left(\right.$ area $\left._{\text {colonies pre-wash }}\right)$, where area is defined by the summed pixel value of every colony on a plate. Finally, we detected colony invasion of the substrate by rubbing the agar surface with a gloved finger under a stream of water to remove any remaining adherent colonies, allowing plates to dry, and acquiring a third image from which invasion was calculated as the (area $\left.a_{\text {hyphal agar invasion }}\right) /\left(\right.$ area $_{\text {colonies pre-wash }}$ ) (Fig. 1a, orange). This imaging approach for $23 \mathrm{C}$. albicans strains produced a robust dataset of approximately 85 million data points for this species across these three biofilm-related phenotypes (Fig. 1b).
Candida filamentation is largely influenced by the nutritional environment

C. albicans isolates displayed a wide range of filamentation phenotypes across solid media (Fig. 2a, b), ranging from profuse filamentation (P76067-Spider medium at $30^{\circ} \mathrm{C}$ ) to a complete absence of radial filaments (529L-YPD at $30^{\circ} \mathrm{C}$ or $37^{\circ} \mathrm{C}$ ). Such differential responses between isolates across environments suggests a complex interplay between the assay conditions used and each genetic background. For example, P94015 produced a strong radial filamentation response on solid Spider medium at $30^{\circ} \mathrm{C}$, despite lacking the major filamentous activator EFG1 and not filamenting on YPD at $37^{\circ} \mathrm{C}$ (Fig. 2C, d, S1). Interestingly, media 
but not temperature strongly influenced filamentation across all $C$. albicans isolates (Fig. 2c-e, Table S1). Specifically, Lee's medium did not elicit strong radial filamentation whereas multiple strains grown on YPD and Spider media formed abundant hyphae at both $30^{\circ} \mathrm{C}$ and $37^{\circ} \mathrm{C}$. Elevated temperature had a variable effect of increasing or decreasing filamentation that was isolate-specific and lacked any clear trends.

To identify isolate characteristics that may associate with filamentation, strains were ordered by their averaged filamentation score for each condition. Rank order plots show most strains filament similarly within each condition (Fig. S2). Surprisingly, the oral isolate $529 \mathrm{~L}$, which has been previously described as failing to filament under most conditions ${ }^{55}$, clustered towards the more robust responses on Lee's and Spider media at $30^{\circ} \mathrm{C}$ and $37^{\circ} \mathrm{C}$ (Fig. S2). This was not a consequence of its clade or body site of isolation, as neither factor correlated with filamentation among these strains (Fig S3).

Ten non-albicans Candida species (NACS) were included in this study to test the utility of this approach beyond C. albicans and define their phenotypic responses ${ }^{53}$. In general, NACS displayed weaker filamentation responses than $C$. albicans and only formed radial hyphae when grown on Spider medium (Fig. 2a-d). Similar to C. albicans, only media shaped NACS filamentation (Table S1). Importantly, some Candida species proved incapable of growing under these conditions. Debaryomyces hansenii did not grow at $37^{\circ} \mathrm{C}$, consistent with its primary identity as an environmental fungus ${ }^{56}$, and Lee's medium was unable to support the growth of C. glabrata and C. lusitaniae. These species were therefore removed from further investigation.

Previous iterations of the $M$ score to measure $C$. albicans filamentation have included relative quantification of colony center wrinkling ${ }^{37,44}$. Among these $23 \mathrm{C}$. albicans isolates and seven NACS, only SC5314 colonies showed prominent wrinkling under any condition (Fig. S4). Therefore, this morphological response is a unique attribute of the SC5314 background that is either much less frequent or entirely absent in other Candida isolates.

\section{Candida filamentation responses are not strongly correlated between solid and liquid substrates}

The substrate context of identical media (i.e., solid or liquid) can alter filamentation responses of isogenic strains ${ }^{57,58}$. To determine if filamentation phenotypes are similar in liquid and solid agar media, six C. albicans isolates from different clades were grown as overnight liquid cultures in YPD at $30^{\circ} \mathrm{C}$, inoculated into four fresh liquid media (Lee's, Spider, YPD, and RPMI 1640) at $30^{\circ} \mathrm{C}$ or $37^{\circ} \mathrm{C}$, imaged after one and four hours, and scored for the frequency of yeast and hyphae in each image using detection scripts developed for single cell analysis (Fig. S5). Filamentation responses in YPD medium at $30^{\circ} \mathrm{C}$ correlated significantly with four of the 16 tested liquid filamentation responses although none were similar solid medium conditions (YPD, $30^{\circ} \mathrm{C}$ ). In fact, Spider medium at $30^{\circ} \mathrm{C}$ was the only condition to elicit similar filamentation responses in liquid and solid substrates (Fig. S6, S7). This suggests that the context of the medium and temperature are important components to activating filamentation responses [59].

\section{Rich nutrient conditions promote Candida species adhesion}

Substrate adherence is the critical initial step during biofilm formation to establish a focal point of cellular aggregation and hyphal initiation ${ }^{25}$. To assay isolate adherence, a constant stream of water was applied to colonies on the agar plates used to quantify filamentation, and their ability to remain bound was measured. This set of $C$. albicans isolates displayed highly variable adherence that was largely dependent on media (Fig. 3a, b, S2, Table S1). While most strains remained adhered to YPD agar at both $30^{\circ} \mathrm{C}$ and $37^{\circ} \mathrm{C}$ (Fig. 3C, d, S8), adherence on Lee's and Spider media varied widely for C. albicans isolates (e.g., P76055 v. P94015 on Spider medium at $37^{\circ} \mathrm{C}$; Fig. 3b, d). In fact, P94015 displayed the strongest adherence of any strain across all assayed conditions (Fig. S8). Adherence was independent of colony size; some isolates, producing small colonies, remained tightly bound to the agar while others, forming large colonies, could be completely washed away (Fig. 3a, b). Surprisingly, adherence decreased at higher temperatures across all three media types (Table S1), but this temperature-dependent regulation was much weaker than the influence of media (Fig. 3e).

Adhesion of NACS was also largely dependent on media. As with C. albicans, most species adhered to YPD but not Spider or Lee's media, but no association was found with temperature (Fig. 3a-d). In a number of cases, NACS adhesion was stronger than that observed for C. albicans isolates (e.g., YPD at $37^{\circ} \mathrm{C}$ ). Principal component analysis (PCA) of adhesion highlighted these differences between C. albicans strains and NACS. Most C. albicans isolates clustered together, whereas NACS occupied a large segment of the two-component space (Fig. 3f), highlighting their relatively wide range of adherence phenotypes.

\section{C. albicans and NACS differ in agar invasion}

To assess agar invasion, all remaining adherent colonies were removed with a gloved finger under running water, and the imprints into the agar from colony invasion were imaged. Conditions that did not produce robust filamentation (Lee's medium) also failed to induce invasion with the exception of P94015 and SC5314, which strongly invaded the agar substrate on Lee's medium at $30^{\circ} \mathrm{C}$ despite neither having produced radial hyphae (Fig. 4a-d, S9). In fact, P94015, the isolate lacking the filamentation activator $E F G 1$, invaded the agar proficiently across all tested conditions (Fig. 4c, d, S2). As with the other biofilmrelated phenotypes, isolates produced a range of invasion profiles from a complete lack of agar invasion to strongly invasive across media and temperature (e.g., P87 and 529L; Fig. 4a-d). Similar to filamentation, media significantly altered colony invasion (Fig. 4e, Table S1), but temperature did not. Yet, in contrast to the other phenotypes, $C$. albicans isolates exhibited a continuum of invasion phenotypes across most media instead of a cluster of phenotypically similar strains with a handful of outliers (Fig. S3).

Unlike most C. albicans isolates, NACS invaded the agar media proficiently across all tested conditions, including Lee's media at $30^{\circ} \mathrm{C}$ and $37^{\circ} \mathrm{C}$ (Fig. $4 \mathrm{a}-\mathrm{d}$ ). This difference between C. albicans and NACS is particularly evident on PC1 of an ordination plot that separates most $C$. albicans isolates from the NACS, with the exception of the invasive strains P94015 and SC5314 (Fig. 4f).

\section{Candida albicans biofilm formation is a multifactorial process}

To compare biofilm-related phenotypes of $C$. albicans isolates to total biofilm production under the same media and temperature conditions, we seeded cells into 96-well plates and allowed biofilms to form in Lee's, Spider, and YPD media at $30^{\circ} \mathrm{C}$ and $37^{\circ} \mathrm{C}$ for $24 \mathrm{~h}^{59}$. RPMI 1640 medium supplemented with $10 \%$ FBS was also included as a common condition to assay biofilm formation. Biofilm production was measured as the optical density $\left(\mathrm{OD}_{600}\right)$ of each well after rinsing to remove non-adherent cells.

The amount of biofilm produced by $C$. albicans was strongly dependent on media and temperature (Fig. 5c). Biofilm mass increased in richer media and was generally greater at $30^{\circ} \mathrm{C}$ than at $37^{\circ} \mathrm{C}$ (Table S1). Thus, increasing the media temperature to $37^{\circ} \mathrm{C}$ led to little biofilm production for almost half of the $\mathrm{C}$. albicans strains in any medium and more stochastic biofilm production among the remaining strains in YPD, which induced the largest biofilms at $30^{\circ} \mathrm{C}$ (Fig. 5a, b, S10). RPMI $1640+10 \%$ FBS (RPMIS) produced a phenotype intermediate to Lee's/Spider media and YPD at both tested temperatures. As with other 

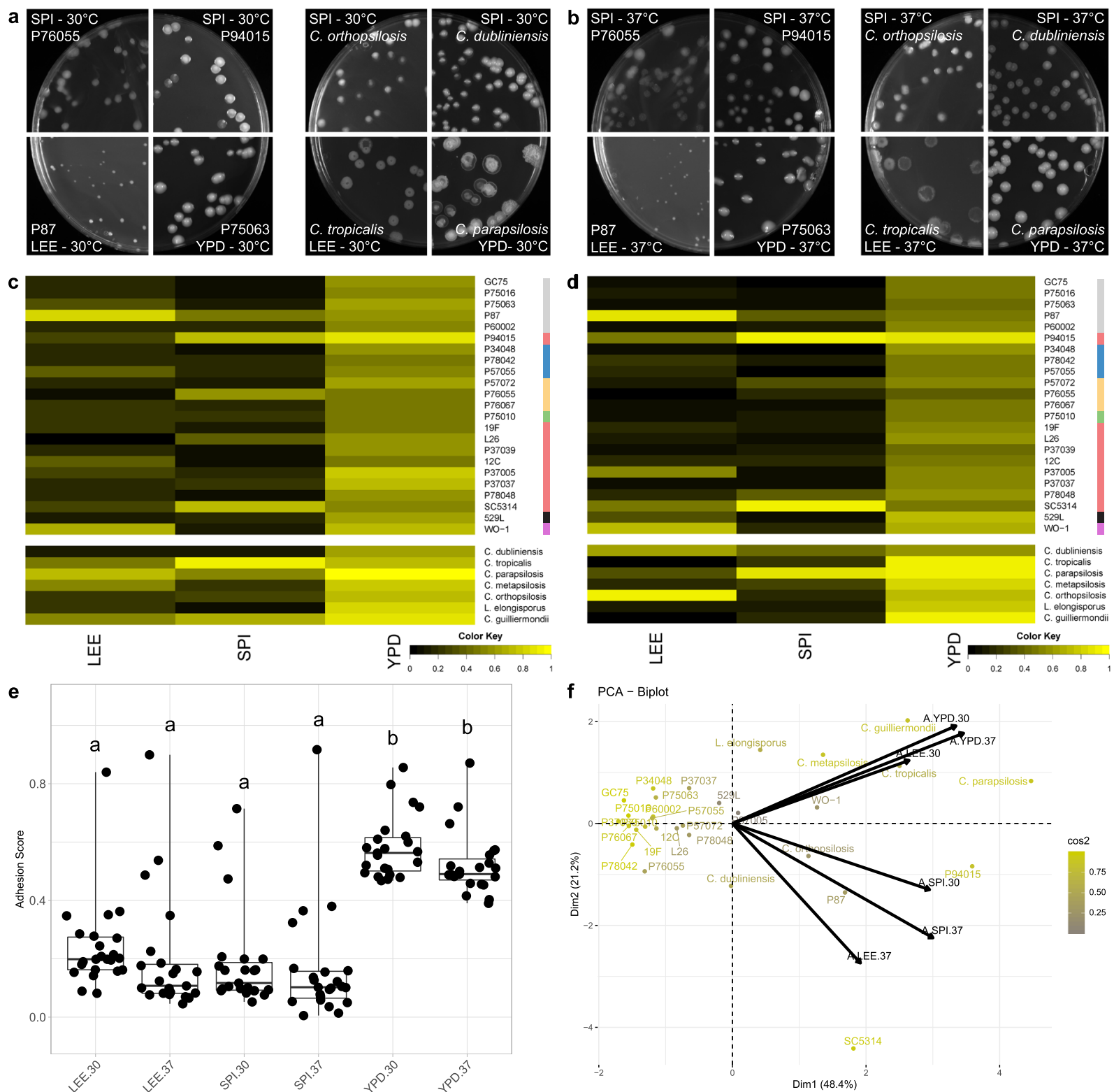

f PCA - Biplot

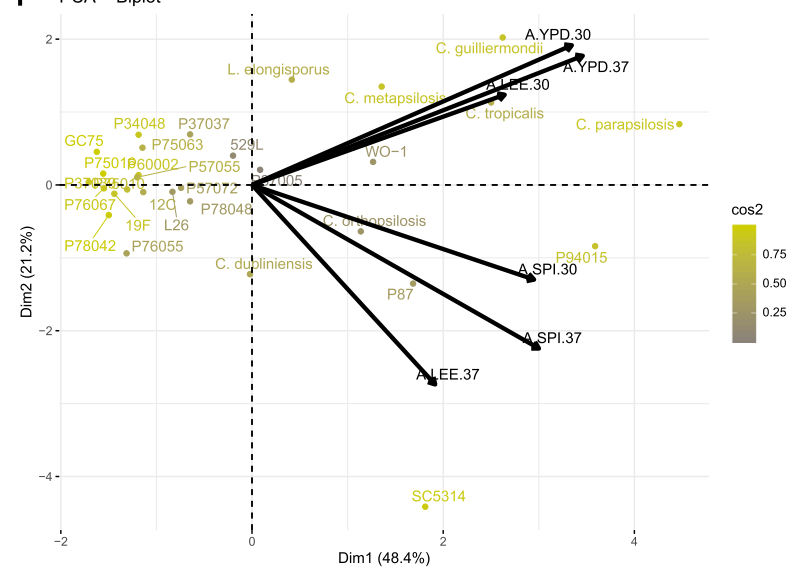

Fig. 3 Candida adhere to substrates in rich media conditions. Plate images of C. albicans isolates (left) and non-albicans Candida species (NACS) (right) highlight the varied adhesion profiles across media at $30^{\circ} \mathrm{C}(\mathbf{a})$ and $37^{\circ} \mathrm{C}$ (b). Heatmaps represent the degree of agar adhesion for Candida

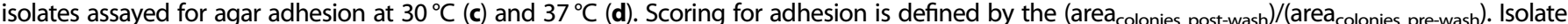
clade is color coded on the right as in Hirakawa et al. ${ }^{37}$. e Agar adhesion scores for each C. albicans isolate were grouped and plotted for each media and temperature combination. Overlaid box plots cover the upper and lower interquartile ranges with the intervening line indicating the mean. Whiskers extend to extreme datapoints. Letters indicate statistically different groups based on Bonferroni-corrected $p$-values $<0.05$ using Dunn's test. f PCA biplots were constructed using adhesion data for all assayed Candida isolates. Coloration from gray to yellow represents the power of the strain in defining PC1 and PC2. Black arrows represent vectorized phenotypic contributions where the arrows are labeled (Phenotype.Media. Temperature). $\mathrm{LEE}=$ Lee's medium, SPI $=$ Spider medium, YPD $=$ Yeast Peptone Dextrose medium.

biofilm-related traits, P94015 and SC5314 displayed aberrant phenotypes (Fig. 5d); P94015 failed to form a biofilm in any condition and SC5314 produced among the most robust biofilms in all tested conditions.

The aggregated data for all strains was plotted as principal components and overlaid with phenotypic vectors for filamentation, adhesion, invasion, and biofilm production (Fig. 6a). Some strains are clearly defined by specific biofilm-related phenotypes that distinguish them from others within this set. For example, P87 resides within the quadrant largely occupied with vectors for adhesion, and P76067 segregates from other strains due to its strong radial filamentation and condition-specific invasion patterns. Importantly, P94015 and SC5314 are clear outliers for biofilm production and its component processes, likely reflecting the combination of aberrant biofilm-related phenotypes compared to most $C$. albicans strains. Invasion vectors tend to reside midway between adhesion and filamentation (Fig. 6a), consistent with the degree of correlations found within these phenotypes (Fig. 6b). Biofilm vectors generally cluster away from the other phenotypes and show no correlation to most biofilm-associated phenotypes (Fig. 6b). Taken together, this indicates that biofilm formation cannot be predicted by any single biofilm-associated phenotype.

To test the integrated contributions of multiple biofilm-related phenotypes to total biofilm production, the relative scores for each component phenotype were weighted equally and summed 

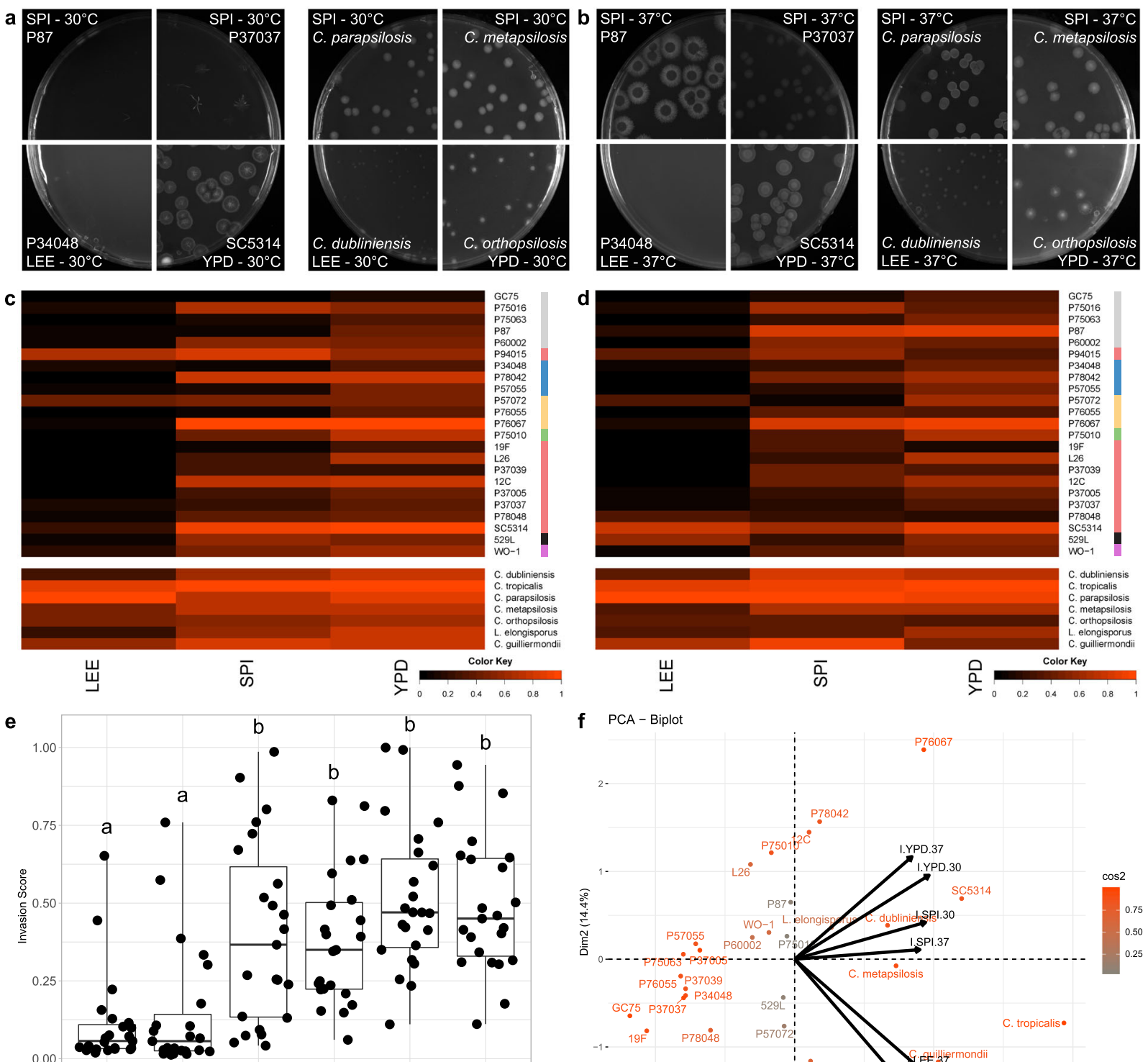

f $\mathrm{PCA}-$ Biplo

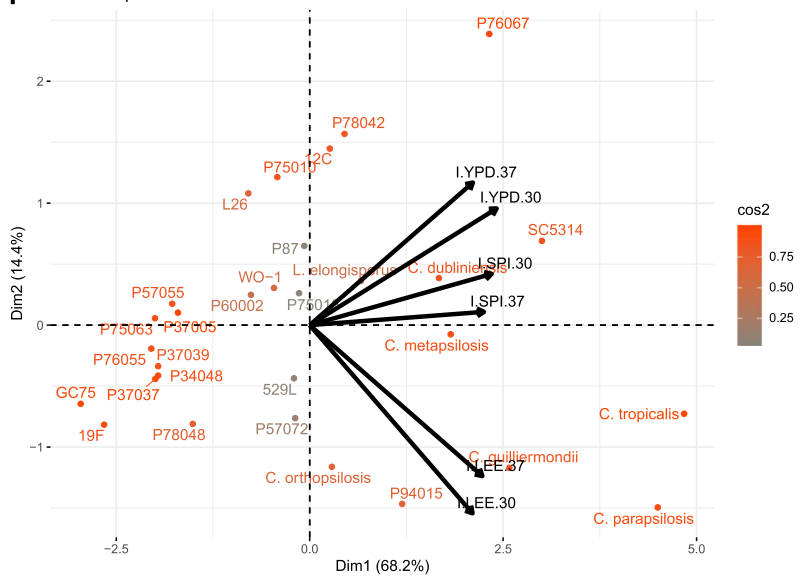

Fig. 4 Candida isolates display high variability in substrate invasion. Plate images of $C$. albicans isolates (left) and non-albicans Candida species (NACS) (right) show resultant invasion profiles across media at $30^{\circ} \mathrm{C}(\mathbf{a})$ and $37^{\circ} \mathrm{C}(\mathbf{b})$. Heatmaps represent the degree of agar invasion for Candida

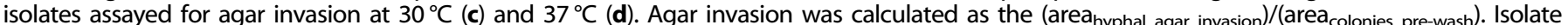
clade is color coded on the right as in Hirakawa et al..$^{37}$. e Agar invasion scores for each C. albicans isolate were grouped and plotted for each media and temperature combination. Overlaid box plots cover the upper and lower interquartile ranges with the intervening line indicating the mean. Whiskers extend to extreme datapoints. Letters indicate statistically different groups based on Bonferroni-corrected $p$-values $<0.05$ using Dunn's test. f PCA biplots were constructed using radial filamentation data for all assayed Candida isolates. Coloration from gray to orange represents the power of the strain in defining PC1 and PC2. Black arrows represent vectorized phenotypic contributions where the arrows are labeled (Phenotype.Media. Temperature). $\mathrm{LEE}=$ Lee's medium, SPI = Spider medium, YPD = Yeast Peptone Dextrose medium.

to generate a composite score that was plotted against biofilm measurements for each media and temperature condition (Fig. 7, Table S2). The composite score for biofilm-related phenotypes and biofilm production in Lee's and Spider media at $37^{\circ} \mathrm{C}$ significantly correlated (Lee's: Pearson's test $=0.48, \mathrm{df}=22, p$-value $=0.02$, Spider: Pearson's test $=0.42, \mathrm{df}=22, p=$ value $=0.04$ ). Weighting individual biofilm-related phenotypes slightly improved but did not substantially change these associations (Fig. S11, Table S3). Thus, the additive contributions of adhesion, filamentation, and invasion are predictive of the degree of biofilm production under some conditions.

\section{DISCUSSION}

Development and implementation of a highly quantitative approach to investigate biofilm-associated processes revealed significant variation among $C$. albicans clinical isolates and nonalbicans Candida species. These responses were largely dependent on the nutrient conditions of the agar substrate. In addition, this variability facilitated construction of an additive model of total biofilm production, where the equivalent contributions of an isolate's biofilm-related phenotypes correlated strongly with biofilm biomass in Lee's and Spider media at $37^{\circ} \mathrm{C}$. Finally, two strains, P94015 and the genome reference strain, SC5314, 

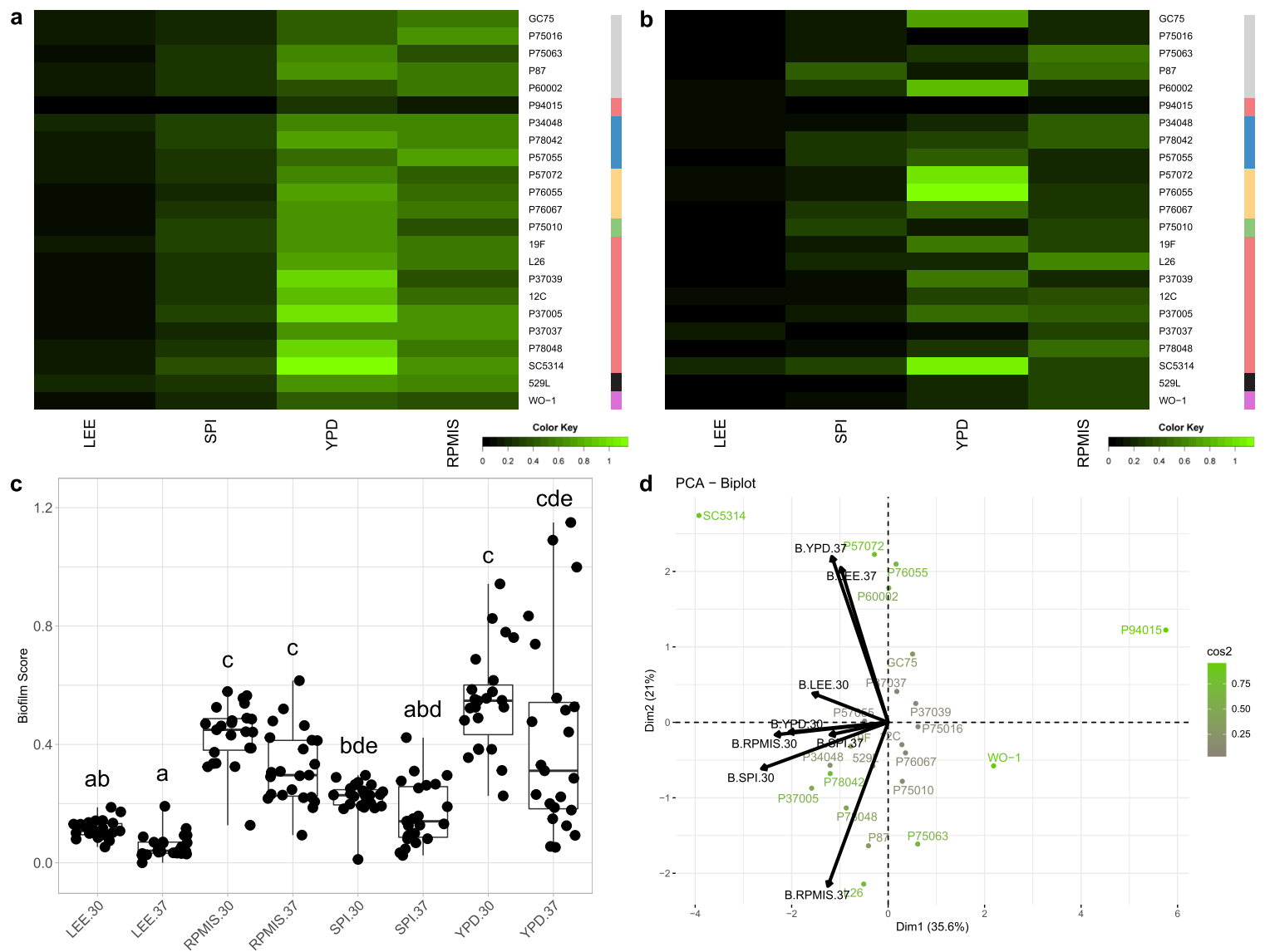

Fig. 5 Biofilm formation increases with media richness across $C$. albicans isolates. Biofilms were seeded in a 96-well high-throughput assay and measured by $\mathrm{OD}_{600}$ after $24 \mathrm{~h}$. Heatmaps represent the total biofilm biomass for C. albicans isolates at $30^{\circ} \mathrm{C}$ (a) and $37^{\circ} \mathrm{C}$ (b). Isolate clade is color coded on the right as in Hirakawa et al. ${ }^{37}$. c Biofilm formation scores for each $C$. albicans isolate were grouped and plotted for each media and temperature combination. Overlaid box plots cover the upper and lower interquartile ranges with the intervening line indicating the mean. Whiskers extend to the extreme data points. Letters indicate statistically different groups (Bonferroni-corrected $p$-values $<0.05$ ) using Dunn's test. d PCA biplots were constructed using radial filamentation data for all assayed Candida isolates. Coloration from gray to green represents the power of the strain in defining PC1 and PC2. Black arrows represent vectorized phenotypic contributions where the arrows are labeled (Phenotype.Media.Temperature). LEE = Lee's medium, SPI = Spider medium, YPD = Yeast Peptone Dextrose medium. RPMIS = RPMI 1640 supplemented with 10\% FBS.

displayed aberrant phenotypes compared to most $C$. albicans isolates, distinguishing them as outliers in biofilm-associated responses.

The automated approaches developed and implemented here greatly enhance investigation of C. albicans biofilm-related phenotypes. Previous work on Candida adhesion and invasion has been mostly descriptive, with the notable exception of adhesion in microtiter plates for large-scale mutant selection and the $M$ score ${ }^{46,47}$. Even with the iterative developments of the $M$ score to increase the reproducibility, ease, and precision of quantifying filamentation ${ }^{37,44,54}$, the labor and time necessary to perform genome-wide mutant screens or large strain collections for these phenotypes have remained a strong deterrent. The analysis pipelines we developed can greatly facilitate these largescale efforts and remove experimental bias by including all colonies in analysis of 1000s of plate images per hour, shifting the burden of experimentation from quantifying strain phenotypes to the experimental setup required to phenotype strains of interest.

This approach was designed with the goal of being suitable for analysis of all Candida species as NACS have been comparatively understudied for these biofilm-associated phenotypes. All NACS in the Candida paraphyletic group showed weak radial filamentation, robust agar invasion, and mostly consistent adherent properties that were dependent on media type. Yet, biofilms produced by NACS have vastly different structures that, in some cases, do not include hyphal cells ${ }^{20}$. This points to either additional or alternative factors that regulate biofilm regulation within these species and/or the different contributions of biofilm-related phenotypes to the lifestyle and success across Candida species.

Phenotypic variability among isolates is a characteristic feature of C. albicans ${ }^{37,60,61}$ and is further supported by our investigation of biofilm-related phenotypes. Some of this variability is likely due to inactivating mutations in key regulators of filamentation and biofilm formation (e.g., EFG1 in P94015 ${ }^{37}$ ). However, the genetic basis for the majority of this variation remains unknown, suggesting that additional, potentially unknown loci contribute to phenotypic variation among these strains, as was recently reported for biofilm formation ${ }^{34}$. Preliminary analysis of gene expression across $21 \mathrm{C}$. albicans represented in this study found differential expression of genes encoding components of the cell wall and cell membrane when grown under identical conditions that may contribute to these phenotypic differences (manuscript under review). Of particular interest will be the strong adhesion phenotype of P94015, which lacks the hyphal-regulator EFG1, and condition-dependent filamentation by C. albicans isolate $529 \mathrm{~L}$, which had been previously described as being incapable of forming true hyphae ${ }^{55}$.

The nutrient environment in which strains are grown strongly impacts biofilm-related phenotypes. Spider medium, which is not a rich media and provides mannitol as the primary carbon source, 

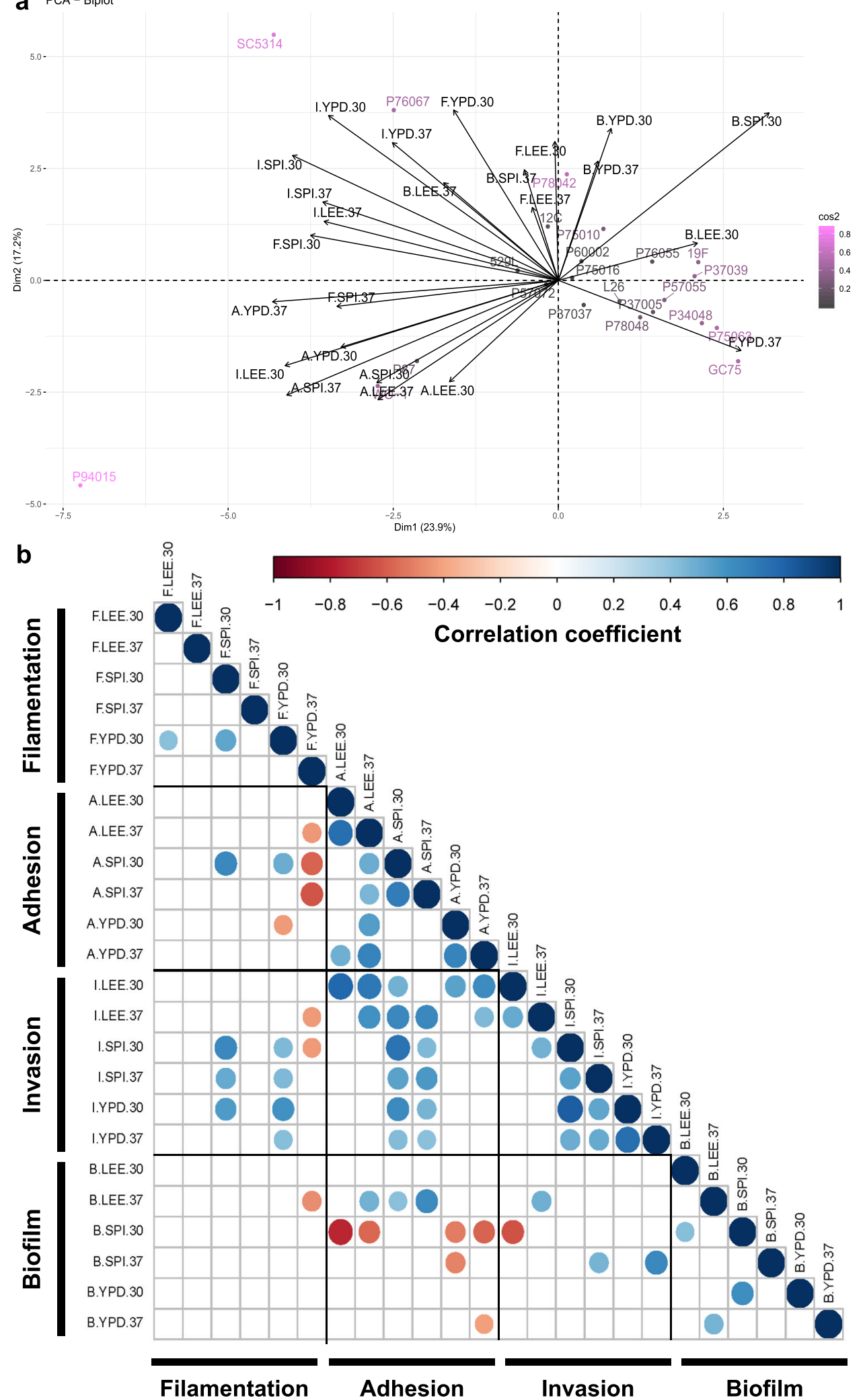

Fig. 6 Most biofilm-associated phenotypes do not correlate. a A PCA biplot was constructed using the aggregate data for C. albicans filamentation, adhesion, invasion, and biofilm production. Coloration of isolate names from gray to orchid indicate the relative contribution of each isolate to determining the coordinate axis. Black arrows indicated vectorized phenotypic contributions where the arrows are labeled (Phenotype.Media.Temperature). b Each biofilm-related phenotype was tested for correlations to all other phenotypes by Pearson's correlation. Blue indicates a significant positive correlation and red indicates a significant negative correlation at an adjusted $p$-value $<0.05$. Phenotypes are labeled as (Phenotype.Media.Temperature). 
a

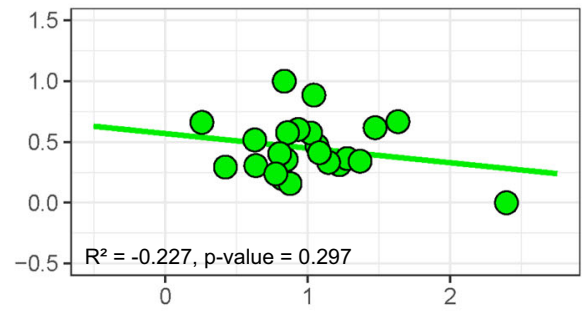

c $\quad \mathrm{SPI} 30^{\circ} \mathrm{C}$

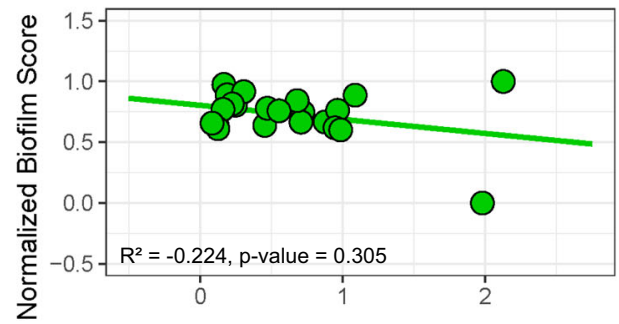

e $\quad$ YPD $30^{\circ} \mathrm{C}$

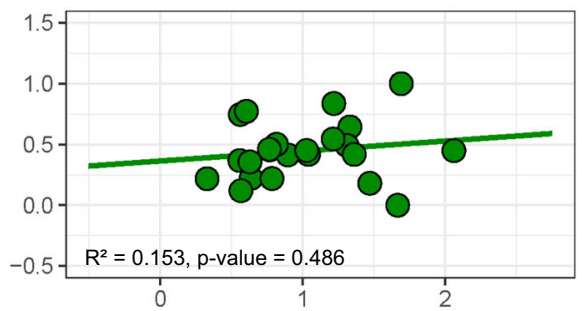

b $\quad \operatorname{LEE} 37^{\circ} \mathrm{C}$

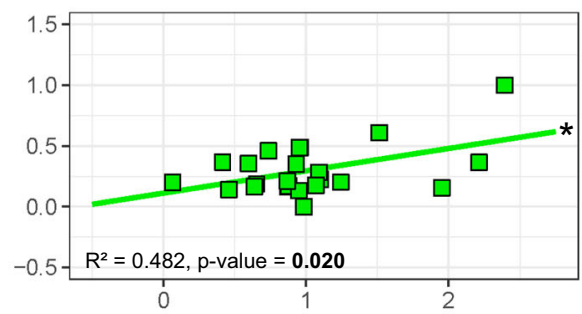

d $\quad \operatorname{SPI} 37^{\circ} \mathrm{C}$

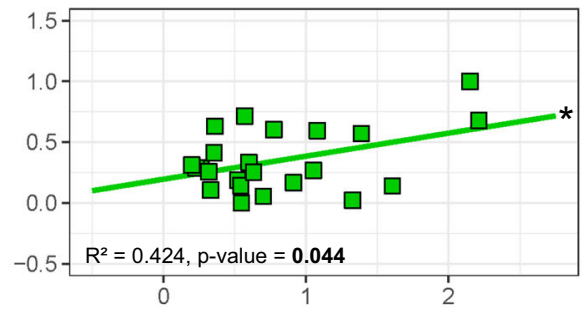

f $\quad \operatorname{YPD} 37^{\circ} \mathrm{C}$

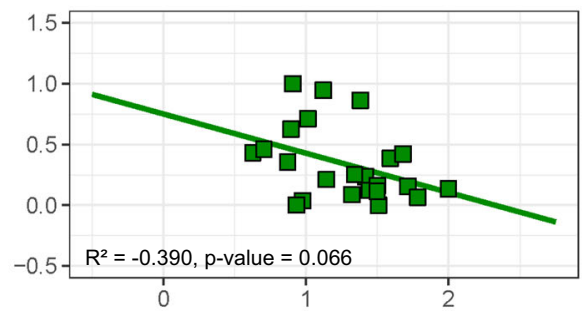

Sum of Normalized Biofilm-related Phenotype Scores

Fig. 7 Cumulative biofilm-related phenotype scores correlate with biofilm production. Normalized filamentation, adhesion, and invasion scores were summed for each tested strain and plotted ( $y$-axis) against the biofilm score ( $x$-axis) for isolates at $30^{\circ} \mathrm{C}($ LEE (a), SPI (c), YPD (e)) and $37^{\circ} \mathrm{C}(\operatorname{LEE}(\mathbf{b})$, SPI (d), YPD (f)).

is commonly used to induce filamentation ${ }^{37,62}$. Indeed, multiple strains display prominent radial filamentation on this medium, but this response is not simply due to the reduced availability of carbon, as growth on amino acid-rich Lee's medium induced little to no hyphal production across Candida species. Yet, in a previous study, Lee's medium proved to be the most common medium to detect filamentation defects when screening a genetic deletion library across ten in vivo filamentation conditions ${ }^{58}$. It is possible that genetic variation in the SC5314 genetic backgrounds or differences in media components could underlie these discrepancies ${ }^{37,63}$. In addition, our data suggests that media content dictates filamentation responses more than growth in liquid or on a solid substrate, even when those contacts activate different genetic pathways ${ }^{58}$. This variation in responses to medium type suggests that use of multiple media in characterizing mutant strains may aid in determining the universal or conditional role of that gene in filamentation and other biofilm-related phenotypes. In addition, recent work highlighting the role of mechanosensing and agar density on microbial invasion and adherence suggests substrate composition may also play a role in Candida biofilm formation ${ }^{64-66}$.

This work also expands beyond filamentation to describe the environmental responses of agar invasion and adhesion. Filamentation and invasion profiles of strains often displayed similar trends, especially between YPD and Spider media (Fig. 6). This may point to similar regulation of hyphal production during the process of biofilm anchoring and generation of aerial hyphae, although these mechanisms likely diverge during the transition to hypoxia as hyphae penetrate further into the substrate ${ }^{67,68}$. Unexpectedly, strains with robust radial projections of hyphae often did not have strong adherence (e.g., P76067). This would suggest that anchoring the biofilm relies on additional activities beyond production of hyphae and their associated repertoire of adhesion molecules ${ }^{25}$. While previous work has noted the dependence of adhesion on media types ${ }^{39}$, trends between rich media and increased adhesion have not been described to the best of our knowledge as was observed here. Finally, this work reveals the surprising result that temperature does not significantly alter the phenotypic outcome of filamentation and invasion using solid agar-based assays. While it is clear that elevated temperature $\left(37^{\circ} \mathrm{C}\right)$ rapidly induces filamentation ${ }^{69,70}$, these assays are commonly conducted in liquid culture with SC5314. Indeed, previous plate-base assays of filamentation showed no clear pattern between hyphal production and temperature ${ }^{37}$. Furthermore, despite a temperature-dependent increase in invasive growth observed for SC5314 in this work, inclusion of clinical isolates that behave differently than the reference strain negated these associations.

Cumulative measures of biofilm-related phenotypes correlated with biofilm production. While these three component phenotypes are known to play important roles in the sequential progression of biofilm maturation, they have not been previously shown to provide predictive power for biofilm potential of individual strains. The lack of correlation under some conditions 
may represent slight differences in sensing solid substrates used between assays (agar v. polystyrene) or additional environmental factors that are not captured in these assays. This is represented in a lack of significant positive correlations between biofilm-related phenotypes and biofilm formation across half of the assayed condition. Yet, composite phenotype scores on Lee's and Spider media at $37^{\circ} \mathrm{C}$ displayed significant positive correlations with production of biofilm. These findings support the importance of each component to total biofilm production and demonstrate that no one phenotype takes clear precedence over another.

As has been observed in other fungal systems ${ }^{37,71}$, the $C$. albicans reference strain displayed multiple phenotypes that distinguished it from other isolates of the same species. For example, center colony wrinkling, previously assessed as a unique variable of plate based filamentation ${ }^{33,37,46}$, is almost exclusive to the genome reference strain SC5314. In general, SC5314 displayed more prominent phenotypes in nearly all conditions, which may reflect lab adaptation or divergent regulation of these biofilm-associated processes prior to isolation. Regardless, we hypothesize that the genetic circuits controlling these processes in SC5314 have diverged from those in other clinical isolates or are expressed at altered levels to produce more robust phenotypes ${ }^{34}$. If SC5314 pathways have been rewired, focused studies only in the genome reference strain may preclude identification of important phenotypic regulators relevant to clinical infections. Indeed, alterations to the organization of biofilmassociated networks recently described within this strain set ${ }^{34,72}$ may indicate this is a common feature of $C$. albicans isolates. Thus, this work further suggests that multiple strain backgrounds should be included in molecular and phenotypic studies of $C$. albicans to ensure similar phenotypes are produced in mutants or under identical conditions.

Among the most vexing questions from this study surround the ability for all of these isolates to cause clinical disease while displaying extensive phenotypic variation of these pathogenic processes. As the majority of these isolates were obtained from systemic infections, how do strains incapable of filamenting strongly under a range of environmental conditions cross epithelial barriers and disseminate across the host? Difficulty recapitulating the precise conditions within the human host through in vitro assays may be one explanation. Alternatively, it may reflect procedures of clinical isolation where a single colony is usually chosen to represent the entire infective population. Thus, the single colony isolate may not accurately embody the clinical population. It is also possible that the site of isolation is not necessarily the site of initial colonization and persistence that would be under stringent selection prior to population expansion. Finally, robust biofilm-associated phenotypes may not be necessary to produce disease in certain patient populations where the lack of innate immune effectors or other competing sources of inflammation allow C. albicans to expand and spread through the host. Investigations focused on the phenotypic diversity found among these $C$. albicans isolates coupled with high-throughput phenotyping will facilitate exploration of the genetic basis for these divergent responses and how massive phenotypic variation can occur for isolates producing similar disease states.

\section{METHODS}

Strains and media used

Strains are listed in Table S4. Altogether, 23 C. albicans clinical isolates ${ }^{37,49-53}$ and ten $\mathrm{NACS}^{53}$ were included in this study. Four media were used for $C$. albicans growth and phenotyping; LEE (Lee's medium) ${ }^{73}$, SPI (Spider medium) ${ }^{74}$, YPD (yeast extract, peptone, dextrose), liquid RPMI 1640 without additives (Corning, Corning, NY), and RPMI 1640 supplemented with $10 \%$ fetal bovine serum.
Filamentation, adhesion, and invasion assays on solid media Overnight cultures for each strain were struck out from $-80^{\circ} \mathrm{C}$ freezer stocks on YPD solid agar. Individual colonies were picked and transferred into $3 \mathrm{~mL}$ of liquid YPD for overnight growth. Overnight cultures were counted using a hemocytometer and diluted into sterile $1 \times$ PBS. Diluted cultures were plated to 100 colonies on LEE, SPI, and YPD solid agar plates. Plates were grown for seven days at $30^{\circ} \mathrm{C}$ or $37^{\circ} \mathrm{C}$ and imaged using a ChemiDoc XRS + imager (Bio-Rad, Hercules, CA) from the top of the plate.

Plate images were scored for radial filamentation and center colony wrinkling using custom scripts described briefly in the detection section below and in detail in the Supplemental Methods. After imaging, each plate was subjected to a flow of water at a $45^{\circ}$ angle for $\sim 3 \mathrm{~s}$ to remove non adherent cells. Flow rate for the adhesion wash was measured at $31.4 \pm 3.6 \mathrm{~mL} / \mathrm{s}$ dispensed from a $1 \mathrm{~cm}$ diameter silicon tube. Plates were allowed to dry and imaged again to score adhesion. Colonies were then directly removed by wiping with a gloved finger under a stream of water and imaged to score for invasion. Adhesion and invasion were also scored using custom visual analysis scripts described below. Each solid plate experiment was conducted in a minimum of six replicates and at least triplicate by two separate researchers.

Detection of radial filamentation, agar adhesion, and agar invasion The following description of quantification is given in greater detail in the Supplemental Methods. Images taken through plate-based assays were processed by the visual analysis tool MIPAR, version 3.0.3 (MIPAR, Worthington, $\mathrm{OH}$ ). The MIPAR recipes [LEE_radial.rcp (Supplemental Data set 1), SPI radial.rcp (Supplemental Data set 2), and YPD radial.rcp (Supplemental Data set 3)] were used to detect filamentation on Lee's, Spider, and YPD media plates. These scripts analyze the image and output a radial filamentation score. Briefly, the plate area was detected, and the edges of the plate were removed to eliminate colonies which contact the edge of the plate. Colonies were then selected within the remaining plate area through a process of smart clustering based on brightness. This clustering step serves the purpose of delineating brightness of the colonies away from the plate coloration. Final radial filamentation was measured as $\left(\right.$ area $_{\text {hyphal growth }}-$ area $\left._{\text {center colonies }}\right) /\left(\right.$ area $\left._{\text {center colonies }}\right)$.

Detection for adhesion was conducted similarly to radial filamentation using the MIPAR script [Adhesion detection.rcp (Supplemental Data set 4)]. Adhesion detection was applied to both the original image (prior to wash) and the washed image. Adhesion was scored as (area colonies post-wash)/

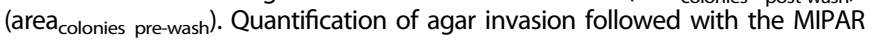
recipe [Invasion_detection.rcp (Supplemental Data set 5)] to identify the remaining hyphal profile from the darker plate background. Invasion detection was applied to both the original image (prior to treatments) and the image of the plate following direct wiping of all colony material from the plate and scored as (area $\left.a_{\text {colonies agar invasion }}\right) /\left(a_{\text {area }}\right.$ colonies pre-wash).

\section{Liquid filamentation and detection}

Overnight cultures of the six C. albicans isolates used for liquid filamentation were grown at $30^{\circ} \mathrm{C}$ in $5 \mathrm{~mL}$ YPD liquid medium. Cultures were separated into $1 \mathrm{~mL}$ aliquots, centrifuged at $5000 \times g$ for $5 \mathrm{~min}$, and washed twice with sterile 1X PBS. Each sample of washed cells was resuspended in $1 \mathrm{~mL}$ of target liquid media (Lee's media, Spider media, YPD, or RPMI), and $100 \mu \mathrm{L}$ of the resuspension was added to $3 \mathrm{~mL}$ of the same media (1:30 dilution). The diluted cultures were then incubated at $30^{\circ} \mathrm{C}$ or $37^{\circ} \mathrm{C}$, shaking at $225 \mathrm{rpm}$ for 1 or $4 \mathrm{~h}$. Glass slides were prepared with $10 \mu \mathrm{L}$ of each culture and visualized across a minimum of eight random fields of view containing at least eight cells using a Leica DM 750 with an attached Leica MC170HD digital camera (Leica, Wetzlar, Germany). Four biological replicates were performed for each strain in each condition.

Images were processed by the visual analysis tool MIPAR, version 3.0.3 (MIPAR, Worthington, OH). The liquid detection recipe [Liquid detection. rcp (Supplemental Data set 6)] was used to select for darker cells against a lighter background. The total number of cells was then tabulated and binned as true hyphae or non-hyphae, where roundness $<0.43$ was scored as hyphae. MIPAR produced the counts of yeast, hyphae, and total cells for all images within this set. The recipe files designed for these assays are available for download in the supplement, as well as accessible here.

\section{Biofilm assay}

A high-throughput 96-well format biofilm assay was adapted from ${ }^{59}$. Cultures were grown for $17 \mathrm{~h}$ in liquid YPD at $30^{\circ} \mathrm{C}$ on a drum roller. Three microliter of 
overnight culture was added to each well of an untreated F-bottom Cellstar ${ }^{\circledR}$ 96-well plate (Greiner, Monroe, NC) that were preloaded with $197 \mu \mathrm{L}$ of the desired growth media (LEE, SPI, YPD, or RPMIS) at the desired temperature for a starting $\mathrm{OD}_{600}$ of $\sim 0.4$. Plates were sealed using a Breathe-Easy sealing membrane (Sigma-Aldrich, St. Louis, MO) and shaken at $250 \mathrm{rpm}$ at $30^{\circ} \mathrm{C}$ or $37^{\circ} \mathrm{C}$ for $90 \mathrm{~min}$ to allow for cell adherence to the bottom of the well. Media was aspirated off, and each well was rinsed with $200 \mu \mathrm{L}$ of sterile $1 \times$ PBS to remove planktonic and non-adherent cells. Two hundred microliter of desired growth media was then added to each well. Plates were loaded into a Synergy $\mathrm{H} 1$ microplate reader (BioTek, Winooski, VT) and grown for $24 \mathrm{~h}$, shaking at the double orbital fast setting at $30^{\circ} \mathrm{C}$ or $37^{\circ} \mathrm{C}$.

Following biofilm growth, media was aspirated from all wells to remove planktonic cells. Wells in which the biofilm was dislodged were excluded from analysis. $\mathrm{OD}_{600}$ was then scanned in a $5 \times 5$ grid for each well with well edges removed from analysis. Optical density reads for each well were then averaged across two technical replicates to calculate total biofilm formed for each biological replicate. The $\mathrm{OD}_{600}$ from wells lacking cells was then subtracted from this average to calculate the average and standard deviation for each strain under each condition. Four biological replicates were used for each strain in each biofilm-growing condition.

\section{Data analysis and visualization}

Analyses were performed in $\mathrm{R}$ (version 3.3.2). Heatmaps were generated using the R-package 'gplots' with color bars divided into 20 independent segments for visualization. Supplemental figure bar charts were generated using the R-package 'ggplot2'. PCA visualization and analysis was conducted using the R-package 'factoextra' where biplots were generated to show the relative contribution of each phenotype to the primary PCs. Detailed protocols for image detection, scoring, and weighting have been included in the Supplemental Methods.

\section{Reporting summary}

Further information on research design is available in the Nature Research Reporting Summary linked to this article.

\section{DATA AVAILABILITY}

The datasets generated during and/or analyzed during this study are available from the corresponding author on reasonable request. Summary of these datasets are included in the published article.

\section{CODE AVAILABILITY}

Code used for data organization and analysis is available through OSU git repositories: https://code.osu.edu/fillinger.22/MIPAR_FWAIBL_Sorted_Score_Files, https://code.osu.edu/fillinger.22/MIPAR_FWAIBL_Normalized_Figures, https://code. osu.edu/fillinger.22/MIPAR_FWAIBL_R2R_Additive_Correlations, and https://code. osu.edu/fillinger.22/mipar_fwaibl-r2r-weighted_phenotypes.

Received: 24 April 2020; Accepted: 11 September 2020; Published online: 09 October 2020

\section{REFERENCES}

1. Davey, M. E. \& O'toole, G. A. Microbial biofilms: from ecology to molecular genetics. Microbiol. Mol. Biol. Rev. 64, 847-867 (2000).

2. de Carvalho, C. C. C. R. Biofilms: Microbial Strategies for Surviving UV Exposure. In Ultraviolet Light in Human Health, Diseases and Environment (ed. Ahmad, S. I.) 233-239 (Springer International Publishing, 2017).

3. Hall-Stoodley, L., Costerton, J. W. \& Stoodley, P. Bacterial biofilms: from the natural environment to infectious diseases. Nat. Rev. Microbiol. 2, 95-108 (2004).

4. Harrison, J. J., Ceri, H. \& Turner, R. J. Multimetal resistance and tolerance in microbial biofilms. Nat. Rev. Microbiol. 5, 928-938 (2007).

5. Stoodley, P., Sauer, K., Davies, D. G. \& Costerton, J. W. Biofilms as complex differentiated communities. Annu. Rev. Microbiol. 56, 187-209 (2002).

6. Katragkou, A. et al. Interactions between human phagocytes and Candida albicans biofilms alone and in combination with antifungal agents. J. Infect. Dis. 201, 1941-1949 (2010).

7. Xie, Z. et al. Candida albicans biofilms do not trigger reactive oxygen species and evade neutrophil killing. J. Infect. Dis. 206, 1936-1945 (2012).
8. Mitchell, K. F., Zarnowski, R. \& Andes, D. R. Fungal super glue: the biofilm matrix and its composition, assembly, and functions. PLoS Pathog. 12, e1005828 (2016).

9. Domenech, M., Ramos-Sevillano, E., García, E., Moscoso, M. \& Yuste, J. Biofilm formation avoids complement immunity and phagocytosis of Streptococcus pneumoniae. Infect. Immun. 81, 2606-2615 (2013).

10. Davies, D. Understanding biofilm resistance to antibacterial agents. Nat. Rev. Drug Discov. 2, 114-122 (2003).

11. Karatan, E. \& Watnick, P. Signals, regulatory networks, and materials that build and break bacterial biofilms. Microbiol. Mol. Biol. Rev. 73, 310-347 (2009).

12. Jamal, M. et al. Bacterial biofilm and associated infections. J. Chin. Med. Assoc. 81, 7-11 (2018).

13. Fox, E. P. \& Nobile, C. J. A sticky situation: untangling the transcriptional network controlling biofilm development in Candida albicans. Transcription 3, 315-322 (2012).

14. Montelongo-Jauregui, Daniel et al. Contributions of Candida albicans dimorphism, adhesive interactions, and extracellular matrix to the formation of dualspecies biofilms with Streptococcus gordonii. MBio 10, 1-15 (2019).

15. Akpan, A. \& Morgan, R. Oral candidiasis. Postgrad. Med. J. 78, 455-459 (2002).

16. Nucci, M. \& Anaissie, E. Revisiting the source of candidemia: skin or gut? Clin. Infect. Dis. 33, 1959-1967 (2001).

17. Sardi, J. C. O., Scorzoni, L., Bernardi, T., Fusco-Almeida, A. M. \& Mendes Giannini, M. J. S. Candida species: current epidemiology, pathogenicity, biofilm formation, natural antifungal products and new therapeutic options. J. Med. Microbiol. 62, 10-24 (2013).

18. Horn, D. L. et al. Epidemiology and outcomes of Candidemia in 2019 patients: data from the prospective antifungal therapy alliance registry. Clin. Infect. Dis. 48, 1695-1703 (2009).

19. Kojic, E. M. \& Darouiche, R. O. Candida infections of medical devices. Clin. Microbiol. Rev. 17, 255-267 (2004).

20. Cavalheiro, M. \& Teixeira, M. C. Candida biofilms: threats, challenges, and promising strategies. Front. Med. 5, 1-15 (2018).

21. Wisplinghoff, $H$. et al. Nosocomial bloodstream infections due to Candida spp. in the USA: species distribution, clinical features and antifungal susceptibilities. Int. J. Antimicrob. Agents 43, 78-81 (2014).

22. Ramage, G., Martínez, J. P. \& López-Ribot, J. L. Candida biofilms on implanted biomaterials: a clinically significant problem. FEMS Yeast Res. 6, 979-986 (2006).

23. Nobile, C. I. \& Johnson, A. D. Candida albicans biofilm and human disease. Annu. Rev. Microbiol. 69, 71-92 (2016).

24. Soll, D. R. \& Daniels, K. J. Plasticity of Candida albicans biofilms. Microbiol. Mol. Biol. Rev. 80, 565-595 (2016).

25. Gulati, M. \& Nobile, C. J. Candida albicans biofilms: development, regulation, and molecular mechanisms. Microbes Infect. 18, 310-321 (2016).

26. Hawser, S. P. \& Douglas, L. J. Biofilm formation by Candida species on the surface of catheter materials in vitro. Infect. Immun. 62, 915-921 (1994).

27. Johnson, C. C., Yu, A., Lee, H., Fidel, P. L. \& Noverr, M. C. Development of a contemporary animal model of Candida albicans-associated denture stomatitis using a novel intraoral denture system. Infect. Immun. 80, 1736-1743 (2012).

28. Chandra, J., Kuhn, D. M., Mukherjee, P. K., Hoyer, L. L. \& Ghannoum, M. A. Biofilm formation by the fungal pathogen. Society 183, 5385-5394 (2001).

29. Otoo, H. N., Lee, K. G., Qiu, W. \& Lipke, P. N. Candida albicans Als adhesins have conserved amyloid-forming sequences. Eukaryot. Cell 7, 776-782 (2008).

30. Klotz, S. A. et al. Candida albicans Als proteins mediate aggregation with bacteria and yeasts. Med. Mycol. 45, 363-370 (2007).

31. Kucharíková, S., Tournu, H., Lagrou, K., van Dijck, P. \& Bujdáková, H. Detailed comparison of Candida albicans and Candida glabrata biofilms under different conditions and their susceptibility to caspofungin and anidulafungin. J. Med. Microbiol 60, 1261-1269 (2011).

32. Zarnowski, R. et al. Novel entries in a fungal biofilm matrix encyclopedia. MBio 5, 1-13 (2014).

33. Uppuluri, P. et al. Candida albicans dispersed cells are developmentally distinct from biofilm and planktonic cells. MBio 9, e01338-18 (2018).

34. Huang, M. Y. et al. Circuit diversification in a biofilm regulatory network. PLoS Pathog. 15, e1007787 (2019).

35. Pujol, C., Daniels, K. J. \& Soll, D. R. Comparison of switching and biofilm formation between MTL-homozygous strains of Candida albicans and Candida dubliniensis. Eukaryot. Cell 14, 1186-1202 (2015).

36. Li, X., Yan, Z. \& Xu, J. Quantitative variation of biofilms among strains in natural populations of Candida albicans. Microbiology 149, 353-362 (2003).

37. Hirakawa, M. P. et al. Genetic and phenotypic intra-species variation in Candida albicans. Genome Res. 25, 413-425 (2015).

38. Warenda, A. J., Kauffman, S., Sherrill, T. P., Becker, J. M. \& Konopka, J. B. Candida albicans septin mutants are defective for invasive growth and virulence. Infect. Immun. 71, 4045-4051 (2003).

39. Weerasekera, M. M. et al. Culture media profoundly affect Candida Albicans and Candida tropicalis growth, adhesion and biofilm development. Mem. Inst. Oswaldo Cruz 111, 697-702 (2016). 
40. Romo, J. A. et al. Development of anti-virulence approaches for candidiasis via a novel series of small-molecule inhibitors of Candida albicans filamentation. MBio 8, 1-16 (2017).

41. Islam, A. et al. Mms21: a putative SUMO E3 ligase in Candida albicans that negatively regulates invasiveness and filamentation, and is required for the genotoxic and cellular stress response. Genetics 211, 579-595 (2019).

42. Banerjee, M., Lazzell, A. L., Romo, J. A., Lopez-Ribot, J. L. \& Kadosh, D. Filamentation is associated with reduced pathogenicity of multiple non- albicans candida species. mSphere 4, 1-8 (2019).

43. Tan, X. et al. The role of Candida albicans SPT20 in filamentation, biofilm formation and pathogenesis. PLOS ONE 9, 1-10 (2014).

44. Nobile, C. J. et al. A recently evolved transcriptional network controls biofilm development in Candida albicans. Cell 148, 126-138 (2012).

45. McCall, A. D., Pathirana, R. U., Prabhakar, A., Cullen, P. J. \& Edgerton, M. Candida albicans biofilm development is governed by cooperative attachment and adhesion maintenance proteins. npj Biofilms Microbiomes 5, 1-12 (2019).

46. Noble, S. M., French, S., Kohn, L. A., Chen, V. \& Johnson, A. D. Systematic screens of a Candida albicans homozygous deletion library decouple morphogenetic switching and pathogenicity. Nat. Genet. 42, 590-598 (2010).

47. Lee, J. A., Robbins, N., Xie, J. L., Ketela, T. \& Cowen, L. E. Functional genomic analysis of candida albicans adherence reveals a key role for the Arp2/3 complex in cell wall remodelling and biofilm formation. PLoS Genet. 12, 1-24 (2016).

48. Finkel, J. S. et al. Portrait of Candida albicans adherence regulators. PLoS Pathog. 8, e1002525 (2012).

49. Cuomo, C. A. et al. Genome sequence for Candida albicans Clinical Oral Isolate 529L. Microbiol. Resour. Announc. 8, 20-21 (2019).

50. Rahman, D., Mistry, M., Thavaraj, S., Challacombe, S. J. \& Naglik, J. R. Murine model of concurrent oral and vaginal Candida albicans colonization to study epithelial host-pathogen interactions. Microbes Infect. 9, 615-622 (2007).

51. Jones, T. et al. The diploid genome sequence of Candida albicans. Proc. Natl Acad. Sci. USA 101, 7329-7334 (2004).

52. Van het Hoog, M. et al. Assembly of the Candida albicans genome into sixteen supercontigs aligned on the eight chromosomes. Genome Biol. 8, 1-11 (2007).

53. Butler, G. et al. Evolution of pathogenicity and sexual reproduction in eight Candida genomes. Nature 459, 657-662 (2009).

54. Dunn, M. J., Kinney, G. M., Washington, P. M., Berman, J. \& Anderson, Z. Functional diversification accompanies gene family expansion of MED2 homologs in Candida albicans. PLoS Genet. 1-29 https://doi.org/10.1371/journal.pgen.1007326 (2018).

55. Moyes, D. L. et al. A biphasic innate immune MAPK response discriminates between the yeast and hyphal forms of candida albicans in epithelial cells. Cell Host Microbe 8, 225-235 (2010).

56. Breuer, U. \& Harms, H. Debaryomyces hansenii-an extremophilic yeast with biotechnological potential. Yeast https://doi.org/10.1002/yea.1374 (2006).

57. Noble, S. M., Gianetti, B. A. \& Witchley, J. N. Candida albicans cell-type switching and functional plasticity in the mammalian host. Nat. Rev. Microbiol. 15, 96 (2016).

58. Azadmanesh, J., Gowen, A. M., Creger, P. E., Schafer, N. D. \& Blankenship, J. R. Filamentation involves two overlapping, but Distinct, programs of filamentation in the pathogenic fungus Candida albicans. G3 Genes Genomes Genet. 7, 3797-3808 (2017).

59. Lohse, M. B. et al. Assessment and optimizations of Candida albicans in vitro biofilm assays. Antimicrob. Agents Chemother. 61, 1-13 (2017).

60. Wu, W., Lockhart, S. R., Pujol, C., Srikantha, T. \& Soll, D. R. Heterozygosity of genes on the sex chromosome regulates Candida albicans virulence. Mol. Microbiol. 64, 1587-1604 (2007).

61. Ropars, J. et al. Gene flow contributes to diversification of the major fungal pathogen Candida albicans. Nat. Commun. 9, 2253 (2018).

62. Calera, J. A., Zhao, X. J. \& Calderone, R. Defective hyphal development and avirulence caused by a deletion of the SSK1 response regulator gene in Candida albicans. Infect. Immun. 68, 518-525 (2000).

63. Abbey, D., Hickman, M., Gresham, D. \& Berman, J. High-resolution SNP/CGH microarrays reveal the accumulation of loss of heterozygosity in commonly used Candida albicans strains. G3 Genes Genomes Genet. 1, 523-530 (2011).

64. Kumamoto, C. A. Molecular mechanisms of mechanosensing and their roles in fungal contact sensing. Nat. Rev. Microbiol. 6, 667-673 (2008).

65. Gordon, V. D. \& Wang, L. Bacterial mechanosensing: the force will be with you, always. J. Cell Sci. 132, jcs227694 (2019).

66. Davis-Fields, M. et al. Assaying how phagocytic success depends on the elasticity of a large target structure. Biophys. J. 117, 1496-1507 (2019).

67. Lu, Y., Su, C., Solis, N. V., Filler, S. G. \& Liu, H. Synergistic regulation of hyphal elongation by hypoxia, $\mathrm{CO} 2$, and nutrient conditions controls the virulence of Candida albicans. Cell Host Microbe 14, 499-509 (2013).

68. Synnott, J. M., Guida, A., Mulhern-Haughey, S., Higgins, D. G. \& Butler, G. Regulation of the hypoxic response in Candida albicans. Eukaryot. Cell 9, 1734-1746 (2010).
69. Sudbery, P. E. Growth of Candida albicans hyphae. Nat. Rev. Microbiol. 9, 737-748 (2011).

70. Desai, P. R., van Wijlick, L., Kurtz, D., Juchimiuk, M. \& Ernst, J. F. Hypoxia and temperature regulated morphogenesis in Candida albicans. PLoS Genet. 11, 1-31 (2015).

71. Kvitek, D. J., Will, J. L. \& Gasch, A. P. Variations in stress sensitivity and genomic expression in diverse S. cerevisiae isolates. PLoS Genet. 4, 31-35 (2008).

72. Liang, S.-H. et al. Hemizygosity enables a mutational transition governing fungal virulence and commensalism. Cell Host Microbe 25, 418-431.e6 (2019).

73. Lee, K. L., Buckley, H. R. \& Campbell, C. C. An amino acid liquid synthetic medium for the development of mycellal and yeast forms of candida albicans. Med. Mycol. https://doi.org/10.1080/00362177585190271 (1975).

74. Liu, H., Köhler, J. \& Fink, G. R. Suppression of hyphal formation in Candida albicans by mutation of a STE12 homolog. Science https://doi.org/10.1126/science.7992058 (1994).

\section{ACKNOWLEDGEMENTS}

We thank the entire Anderson lab for helpful discussions and feedback during experiments. We also thank the lab of Dr. Chad Rappleye for comments and critique of experimental approach and visual analysis pipelines. We would also like to thank Dr. Richard Bennett for the C. albicans strains, as well as Dr. Geraldine Butler and Dr. Mike Lorenz for the NACS set used within this study. The visual detection recipes developed here were built with input and support from John Sosa and Pavel Sul of the MIPAR team, and we thank them for their continued support of this project. This work was supported by a National Institutes of Health grant (R01Al148788) to M.Z.A. and the American Heart Association grant AHA 20PRE35200201, M.J.D., 2020. R.J.F. was supported by an NIH F31 fellowship (1F31DE029409-01).

\section{AUTHOR CONTRIBUTIONS}

Conceptualization: M.J.D., R.J.F., and M.Z.A. Data curation: M.J.D., R.J.F., and L.M.A. Formal analysis: M.J.D. and R.J.F. Funding acquisition: M.J.D., R.J.F., and M.Z.A. Investigation: M.J.D., R.J.F., and L.M.A. Methodology: M.J.D. and R.J.F. Project administration: M.Z.A. Resources: M.Z.A. Software: M.J.D. and M.Z.A. Supervision: M. Z.A. Validation: M.J.D., R.J.F., and M.Z.A. Visualization: M.J.D., R.J.F., and L.M.A. Writingoriginal draft: M.J.D. and R.J.F. Writing-review and editing: M.J.D., R.J.F., L.M.A., and M.Z.A. M.J.D. and R.J.F. contributed equally to this work. Together they drew up the study design, developed the method, performed all experiments side-by-side, and equally contributed to the final analysis such that their efforts are considered to be equal.

\section{COMPETING INTERESTS}

The authors declare no competing interests.

\section{ADDITIONAL INFORMATION}

Supplementary information is available for this paper at https://doi.org/10.1038/ s41522-020-00149-5.

Correspondence and requests for materials should be addressed to M.Z.A.

Reprints and permission information is available at http://www.nature.com/ reprints

Publisher's note Springer Nature remains neutral with regard to jurisdictional claims in published maps and institutional affiliations.

Open Access This article is licensed under a Creative Commons Attribution 4.0 International License, which permits use, sharing, adaptation, distribution and reproduction in any medium or format, as long as you give appropriate credit to the original author(s) and the source, provide a link to the Creative Commons license, and indicate if changes were made. The images or other third party material in this article are included in the article's Creative Commons license, unless indicated otherwise in a credit line to the material. If material is not included in the article's Creative Commons license and your intended use is not permitted by statutory regulation or exceeds the permitted use, you will need to obtain permission directly from the copyright holder. To view a copy of this license, visit http://creativecommons. org/licenses/by/4.0/.

(c) The Author(s) 2020 